\title{
Analyzing clinical and genetic aspects of axonal Charcot-Marie-Tooth disease
}

\author{
Hye Mi Kwon ${ }^{\oplus}$ and Byung-Ok Choi*® \\ Department of Neurology, Samsung Medical Center, Sungkyunkwan University School of Medicine, Seoul, Korea
}

\begin{abstract}
Charcot-Marie-Tooth disease (CMT) is the most common hereditary motor and sensory peripheral neuropathy. CMT is usually classified into two categories based on pathology: demyelinating CMT type 1 (CMT1) and axonal CMT type 2 (CMT2) neuropathy. CMT1 can be distinguished by assessing the median motor nerve conduction velocity as greater than $38 \mathrm{~m} / \mathrm{s}$. The main clinical features of axonal CMT2 neuropathy are distal muscle weakness and loss of sensory and areflexia. In addition, they showed unusual clinical features, including delayed development, hearing loss, pyramidal signs, vocal cord paralysis, optic atrophy, and abnormal pupillary reactions. Recently, customized treatments for genetic diseases have been developed, and pregnancy diagnosis can enable the birth of a normal child when the causative gene mutation is found in CMT2. Therefore, accurate diagnosis based on genotype/phenotypic correlations is becoming more important. In this review, we describe the latest findings on the phenotypic characteristics of axonal CMT2 neuropathy. We hope that this review will be useful for clinicians in regard to the diagnosis and treatment of CMT.
\end{abstract}

Key words: Charcot-Marie-Tooth disease, Classification, Mutation, Gene, Diagnosis.

\section{Introduction}

With the recent rapid development of science and technology, the diagnosis rate of genetic diseases has gradually increased compared to the past. In addition, whole exome sequencing (WES) and whole genome sequencing (WGS) are now used for diagnosis, and there is a growing interest in rare genetic diseases. Promising progress is being made in the development of treatments for rare diseases. In particular, therapeutic drugs for rare genetic disorders, including Duchene muscular dystrophy, amyloidosis, and spinal muscle atrophy, have been developed and used in patients. In this review, we describe the latest findings on the phenotypic characteristics of axonal Charcot-Marie-Tooth
(CMT) neuropathy type 2 .

CMT is the most common rare genetic disease and is a clinically and genetically heterogeneous disease. To date, more than 130 causative genes for CMT have been reported. On the basis of nerve pathology, CMT is divided into two categories: demyelinating and axonal neuropathy $[1,2]$. Demyelinating and axonal CMT neuropathy can be generally distinguished by assessing the median motor nerve conduction velocity (NCV), which is lower and higher than $38 \mathrm{~m} / \mathrm{s}$, respectively [1,2]. Axonal-type CMT represents approximately one-third of the total CMT [3-5], and shows unusual clinical features, such as delayed motor development, hyperreflexia, ankle contracture, pyramidal track sign, vocal cord paralysis, hearing loss, optic atrophy, abnormal pupillary

\footnotetext{
Received: 24 May 2021, Revised: 14 July 2021, Accepted: 3 September 2021, Published: 31 December 2021

*Corresponding author: Byung-Ok Choi, M.D., Ph.D. (iD https://orcid.org/ 0000-0001-5459-1772

Department of Neurology, Samsung Medical Center, Sungkyunkwan University School of Medicine, 81 Irwon-ro, Gangnam-gu, Seoul 06351, Korea.

Tel: +82-2-3410-1296, Fax: +82-2-3410-0052, E-mail: bochoi@skku.edu

Conflict of interest: The authors declare that they do not have any conflicts of interest.

(c) This is an open-access article distributed under the terms of the Creative Commons Attribution Non-Commercial License (http://creativecommons.org/licenses/by-nc/4.0/) which permits unrestricted non-commercial use, distribution, and reproduction in any medium, provided the original work is properly cited.

(c) Copyright 2021 by the Korean Society of Medical Genetics and Genomics 
reaction, and kyphoscoliosis [6-17].

Until now, the causative gene mutation underlying CMT could be identified in only $60 \%$ to $70 \%$ of all CMT patients, while that in the others could not be identified even by using WES. Therefore, the WGS test is needed to investigate the causative genes of those that are remaining. The cause of CMT may be a regulatory gene in the intron, a splicing mutation, copy number variation, structural variation, or transposal element. The classification of axonal CMT2 neuropathy may be more complicated than if all causative gene mutations are known.

Preimplantation genetic diagnosis (PGD) can be performed if the causative gene mutation is found in CMT2 patients. It is a great blessing to patients and their families to enable the birth of a normal child through PGD in CMT2, for which there is currently no cure. Thus, accurate diagnosis based on genotype/ phenotype correlation is essential in CMT2, and PGD is, therefore, widely used in CMT2 patients. Here, we intend to present a comprehensive review which would be helpful to physicians and researchers majoring in medical genetics by investigating the latest updated contents of CMT2 and describing the phenotypic characteristics associated with it.

\section{Charcot-Marie-Tooth Disease Type 2}

\section{CMT2A}

\section{1) $\mathrm{CMT} 2 \mathrm{~A} 1$}

CMT2A1 arises from a heterozygous mutation in the kinesin family member $1 \mathrm{~B}$ (KIF1B) gene on chromosome $1 \mathrm{p} 36$ [18] and has an autosomal dominant inheritance pattern (Table 1). One family with a KIF1B mutation was identified. CMT2A1 can occur from childhood to 50 years of age. It is accompanied by distal muscle weakness, pes cavus, and muscle atrophy in the lower extremities. Distal sensory impairment, hyporeflexia, and steppage gait have also been observed. CMT2A1 is typically associated with slightly reduced motor NCV. A sural nerve biopsy can reveal axonal atrophy, axon degeneration/regeneration, reduced myelinated fibers, and small onion bulb formation.

\section{2) $\mathrm{CMT} 2 \mathrm{~A} 2 \mathrm{~A}$}

CMT2A2A involves a defect in the MFN2 gene on chromosome 1p36.2 [19-21]. Both mitochondrial arrangement and size differ depending on the physiologic condition, pathological state, and cell type. MFN2 mediates mitochondrial fusion and dynamic balance, and CMT2A2A has autosomal dominant inheritance. CMT2A2A occurs widely in children and older adults (aged 50 or more years), with a slow progression. Early onset of CMT2A2A shows fatal subacute encephalopathy and has been associated with increased severity. However, up to $25 \%$ cases of CMT2A2A are asymptomatic or mildly affected. This indicates incomplete penetrance. Distal muscle weakness and sensory impairments may occur. Symptoms usually begin in the lower limbs and finally progress to the upper limbs. The other symptoms are pes cavus, foot deformities, and hammer toes. Pyramidal signs, extensor plantar responses, increased muscle tone, or deep tendon reflexes are rarely observed. Fatal subacute encephalopathy and cognitive decline are rare in the central nervous system (CNS). Spasticity and pyramidal features have also been reported. In the early stages, scoliosis and skeletal contractures can occur, while optic atrophy and hearing loss are less likely. Motor NCV is normal or slightly decreased. In the sural nerve biopsy, reduced myelinated fibers and small onion bulb formations can be seen.

\section{3) $\mathrm{CMT} 2 \mathrm{~A} 2 \mathrm{~B}$}

CMT2A2B is caused by either a homozygous or a compound heterozygous mutation in the MFN2 gene on chromosome 1p36.2. CMT2A2B has an autosomal recessive inheritance pattern [22]. The onset of CMT2A2B occurs within the first year of life. CMT2A2B shows a very wide range of severity, but most patients may have to use a wheelchair at a later stage of the disease. The common symptoms of CMT2A2B are distal muscle weakness, sensory impairment, gait disturbance, and pes cavus. Scoliosis and proximal muscle weakness may also occur. Delayed motor development is frequently observed. Ophthalmologic features including pale optic discs and optic nerve atrophy have also been observed, and visual impairment occurs at a later age. Some patients may develop hearing impairments. Sural nerve biopsy may reveal a reduced number of large myelinated fibers.

\section{CMT2B}

\section{1) $\mathrm{CMT} 2 \mathrm{~B}$}

CMT2B is caused by a heterozygous mutation in $R A B 7 A$, a member of the RAS oncogene family (RAB7A) gene on chromosome 3q21 [23], which encodes small GTPases, ubiquitously expressed proteins that play a role in the regulation of trafficking, maturation, and fusion of endocytic and autophagic vesicles. CMT2B has an autosomal dominant inheritance. Most CMT2B cases occur in the second decade of life and usually start in the lower limbs. It eventually progresses to the upper limbs. CMT2B overlaps clinical features with hereditary sensory and autonomic neuropathy type I (HSAN1). Muscle weakness and atrophy in 


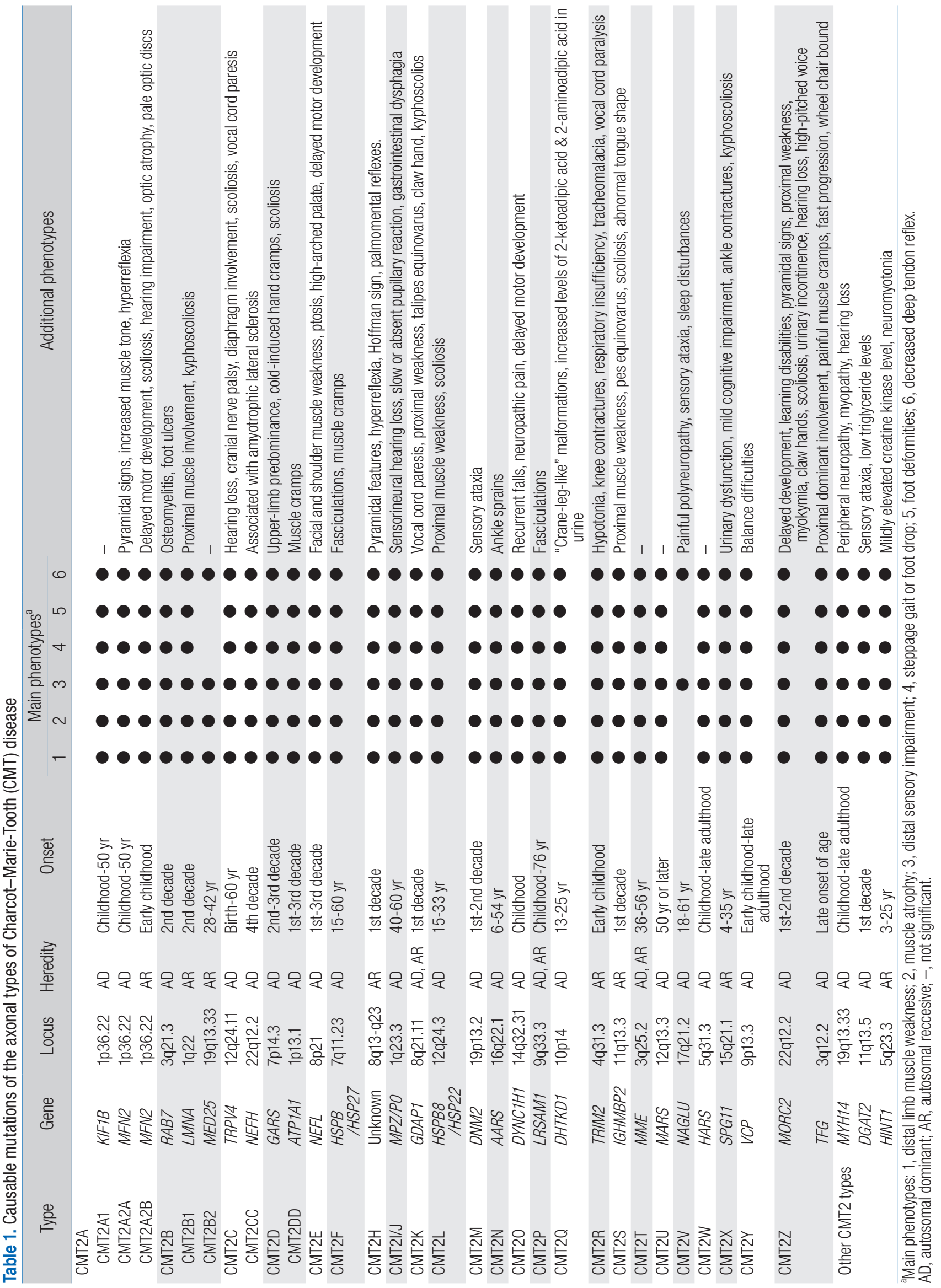


the lower limbs, distal sensory impairment, steppage gait, and decreased deep tendon reflexes can occur. Hammer toes, pes planus, and pes cavus may also be observed in the lower limbs. In addition, ulcers in the distal limbs caused by sensory neuropathy often lead to infection. Sometimes, autoamputation is necessary. Moreover, osteomyelitis or necrosis can occur. Motor NCV decreases slightly. In the sural nerve biopsy, reduced myelinated fibers and small onion bulbs have been observed. Axonal atrophy and degeneration and regeneration were also observed.

\section{2) $\mathrm{CMT} 2 \mathrm{~B} 1$}

CMT2B1 is caused by a homozygous mutation in the lamin A/ $\mathrm{C}(L M N A)$ gene on chromosome 1q22 [24]. CMT2B1 has an autosomal recessive inheritance pattern. The $L M N A$ gene encodes lamin A and lamin C. Lamins are the structural protein components of the nuclear lamina, a network of proteins underlying the inner nuclear membrane that determines nuclear shape and size. Lamins constitute a class of intermediate filaments, and three types, namely $A, B$, and $C$ have been described in mammalian cells. CMT2B1 starts mainly in the second decade of life and has a severe course. Muscle weakness and atrophy are common symptoms of CMT2B1. In addition, sensory impairment, pes cavus, hypo- or areflexia, and scoliosis may be observed. Small onion bulb formation and decreased myelinated fibers can be found, while axonal atrophy and axon degeneration/regeneration are observed through nerve biopsy.

\section{3) $\mathrm{CMT} 2 \mathrm{~B} 2$}

CMT2B2 is caused by a homozygous mutation in the mediator complex subunit 25 (MED25) gene [25], which encodes a protein belonging to the mediator complex, an evolutionarily conserved multisubunit RNA polymerase II transcriptional regulator complex. CMT2B2 has an autosomal recessive inheritance pattern. CMT2B2 has been reported in only one family member. CMT2B2 begins between the ages of 28 and 42 years. The initial symptoms of CMT2B2 include distal muscle atrophy and weakness in the lower limbs. It eventually progresses to the upper limbs. Motor NCV has been reported to decrease slightly.

\section{3. $\mathrm{CMT} 2 \mathrm{C}$}

\section{1) $\mathrm{CMT} 2 \mathrm{C}$}

CMT2C is caused by a heterozygous mutation in the transient receptor potential cation channel subfamily V member 4 (TRPV4) gene on chromosome $12 q 24[26,27]$. CMT2C is also known as hereditary motor and sensory neuropathy type IIC (HMSN2C) and has an autosomal dominant inheritance pattern. The TRPV4 cation channel mediates calcium influx in response to physical, chemical, and hormonal stimuli in ciliated epithelial cells. The onset age of CMT2C ranges from birth to older adulthood (the age of 60 years). Early onset was reported to be associated with increased severity. It overlaps with the clinical features of distal hereditary motor neuropathy type VII (dHMN VII). This suggests an incomplete penetrance. In the upper and lower limbs, distal muscle weakness and early wasting of the hand muscles, along with impaired manual dexterity are usually reported. Hand weakness is worse with coldness, and proximal limb muscles are affected in severe cases. Additionally, other signs include diaphragm involvement, intercostal muscle involvement, scoliosis, and vocal cord paresis. Similarly, abducens or oculomotor nerve palsy, hearing loss, stridor, intercostal muscle, and diaphragm involvement can occur in this subtype. Urinary incontinence and urgency may be observed in some patients. In some cases, sloping shoulders are observed due to shoulder girdle muscle atrophy. Motor NCV is normal. The sensory nerve action potential (SNAP) and compound muscle action potential (CMAP) amplitudes decrease. Neurogenic atrophy can be observed in muscle biopsy.

\section{2) $\mathrm{CMT} 2 \mathrm{CC}$}

CMT2CC is caused by a heterozygous mutation in the neurofilament heavy (NEFH) gene on chromosome 22q12 [28]. It has an autosomal dominant inheritance pattern. In particular, three mAl neuron-specific intermediate filaments, namely the NEFH, NEFM, and NEFL, are the most prominent cytoskeletal components in large myelinated axons. The onset of CMT2CC varies from early childhood to the fourth decade of life. Two unrelated families with variable severity have been reported. The main features of CMT2CC are distal muscle weakness and muscle atrophy, high-arched feet, gait disturbance, distal sensory impairment, and hyporeflexia. Motor NCV is normal or decreases slightly, and the CMAP amplitude decreases. Neurogenic and myopathic changes have been identified using electromyography (EMG) and muscle biopsy.

\section{CMT2D}

\section{1) $C M T 2 D$}

CMT2D is caused by a mutation in the glycyl-tRNA synthetase (GARS) gene [29], which encodes an enzyme that charges tRNA molecules with glycine through an aminoacylation reaction. CMT2D has an autosomal dominant inheritance pattern. CMT2D 
begins during the second decade of life and progresses slowly. Distal spinal muscular atrophy type 5A (dSMA5A) is an allelic disorder with a similar phenotype [30], but CMT2D presents more severe distal sensory involvement. CMT2D is predominantly characterized by intrinsic muscle weakness in the hand. Other symptoms include cold-induced hand cramps. Normal motor NCV has been observed.

\section{2) CMT2DD}

CMT2DD is caused by a heterozygous mutation in the ATPase $\mathrm{Na}^{+} / \mathrm{K}^{+}$transporting subunit alpha 1 (ATP1A1) gene on chromosome $1 p 13$ [31]. It has an autosomal dominant inheritance pattern. This gene encodes the alpha- 1 isoform of $\mathrm{Na}^{+}, \mathrm{K}^{+}$-ATPase, an integral membrane protein that is responsible for establishing and maintaining the electrochemical gradients of $\mathrm{Na}$ and $\mathrm{K}$ ions across the plasma membrane. The onset variants of CMT2DD are between late childhood and an individual's fifties, but most patients develop symptoms in the second or third decade of life. CMT2DD shows a slow progression, and most patients maintain their ambulatory function, while others have a subclinical course with minimal neurological deficits. The typical features are atrophy of the intrinsic hand muscles, pes cavus, sensory loss, steppage gait, foot drop, and hypo- or areflexia. Muscle cramps are also observed. In addition, the motor NCV is normal. Both the CMAP and SNAP amplitudes are reduced. A sural nerve biopsy showed loss of large myelinated fibers, while regenerating axons and thin myelin were observed.

\section{CMT2E}

CMT2E is caused by a heterozygous mutation in the NEFL gene on chromosome 8p21 [31], which encodes a subunit of type IV intermediate filament heteropolymers, a major component of the neuronal cytoskeleton [32]. It has an autosomal dominant inheritance pattern. The onset of CMT2E begins before the third decade of life. Its severity varies widely. Muscle weakness, distal sensory impairment, steppage gait, and decreased deep tendon reflexes are the main features of CMT2E. In addition, pes cavus, hammer toes, and joint contractures can be found. In severe cases, claw hand deformities have been observed. Weakness of the shoulder or facial muscles, ptosis, and high-arched palate can also be found. A sural nerve biopsy showed primary axonopathy, characterized by giant axons containing disorganized neurofilaments. Fiber size variation, predominance of type 1 or type 2 fibers, internal nuclei, and connective tissue can be found in muscle biopsy. CMT2E patients also exhibited nemaline rods, myofibrillar disruption, and myofiber degeneration.

\section{6. $\mathrm{CMT} 2 \mathrm{~F}$}

CMT disease type $2 \mathrm{~F}$ is caused by a mutation in the heat-shock protein (HSP) family B (small) member 1 (HSPB1) gene encoding heat-shock 27-kD protein 1 (HSPB1) [33]. It has an autosomal dominant inheritance pattern. HSPs belong to a larger group of polypeptides and stress proteins. HSPs are involved in various responses to environmental challenges and developmental transitions. In addition, the synthesis of the small (27-kD) HSP was shown to be correlated with the acquisition of thermotolerance. CMT2F shows muscle weakness and muscle atrophy in the lower limbs, progressing to the upper limbs at a later stage. Distal sensory impairment, pes cavus, foot drop, and hyporeflexia are common, and muscle cramps and fasciculations can be found. In severe cases, claw hand deformities were observed. Finally, chronic axonal neuropathy is manifested through a sural nerve biopsy.

\section{7. $\mathrm{CMT} 2 \mathrm{H}$}

$\mathrm{CMT} 2 \mathrm{H}$ maps to the same region containing the gangliosideinduced differentiation-associated protein 1 (GDAP1) gene [34]. It has an autosomal recessive inheritance pattern. The symptoms of CMT2H begin in early childhood. Limb muscle weakness and muscle atrophy, pes cavus, foot drop, steppage gait, and areflexia may occur. The additional symptoms include pyramidal features, abnormalities in both the brisk Hoffman's and palmomental reflexes, and hyperreflexia in the upper limbs. CMT2H shows a normal motor NCV. In sural nerve biopsy, myelin sheaths are thin and axonal regeneration is rare.

\section{8. СMT2I}

CMT2I is caused by a heterozygous mutation in the myelin protein zero (MPZ) gene on chromosome 1q23 [35,36]. It has an autosomal dominant inheritance pattern and begins between the ages of 40 and 60 years. The first symptoms of CMT2I usually begin in the lower limbs, even when they progress to the upper limbs in a later stage of the disease, given its genetic heterogeneity. Distal muscle and sensory impairment, gait disturbance, and pes cavus can occur in CMT2I patients. The motor NCV is normal or slightly decreased. A sural nerve biopsy showed loss of myelinated fibers and axonal degeneration and regeneration.

\section{9. СMT2」}

CMT2 with hearing loss and pupillary abnormalities is also referred to as CMT2J, which is caused by a heterozygous mutation in the MPZ gene on chromosome 1q23 $[35,37,38]$. CMT2J has an autosomal dominant inheritance pattern. Onset often occurs 
between the fourth and the sixth decade of life. Distal muscle weakness has been observed in the lower limbs, and it extends to the upper limbs. Foot drop, gait disturbance, foot deformities, sensory impairment, and decreased deep tendon reflexes can also occur. The additional symptoms are gastrointestinal dysphagia and sensorineural hearing loss. Both slow pupillary reaction with slow light convergence reflex and absent pupillary reaction can be found in the eyes. Finally, a normal to mildly decreased motor NCV has been observed. Axonal degeneration and regeneration may be observed through nerve biopsy.

\section{CMT2K}

Autosomal recessive axonal CMT disease type $2 \mathrm{~K}$ is caused by either a homozygous or a compound heterozygous mutation in the GDAP1 gene on chromosome 8q [36], which encodes an integral membrane protein of the outer mitochondrial membrane expressed in both the CNS and peripheral nervous system, particularly in Schwann cells. CMT2K has an autosomal dominant inheritance $[39,40]$. The onset age of CMT2K occurs during infancy (three years old). CMT2K shows a more severe course with an earlier onset. It usually begins in the lower limbs, and upper limb is affected during the first decade. This subtype shares the same phenotype as the CMT4A allelic disorder. The main symptoms include distal muscle weakness and atrophy, possible involvement of proximal muscles, distal sensory impairment, absent deep tendon reflexes, kyphoscoliosis, claw hand deformities, and talipes equinovarus. The motor NCV is normal or slightly decreased. However, nerve biopsy may reveal pseudoonion bulb formation, axonal degeneration/regeneration, and reduced myelinated fibers.

\section{CMT2L}

$\mathrm{CMT} 2 \mathrm{~L}$ is caused by a mutation in the heat shock protein family B (small) member 8 (HSPB8) gene [41]. CMT2L has an autosomal dominant inheritance pattern. Distal hereditary motor neuronopathy type $2 \mathrm{~A}$ ( $\mathrm{dHMN} 2 \mathrm{~A}$ ) is an allelic disorder with an overlapping phenotype. The onset age of CMT2L is between 15 and 33 years. Muscle weakness and sensory impairment usually occur in the lower extremities. Proximal muscle involvement is rare. The additional symptoms include scoliosis. Motor NCV is normal, and the SNAP and CMAP amplitudes are decreased. Finally, large myelinated fibers are reduced, and thin myelinated axons can be found through nerve biopsy. EMG shows denervation and fibrillation potentials.

\section{2. СMT2M}

CMT2M is caused by a heterozygous mutation in the dynamin 2 (DNM2) gene on chromosome 19p [42]. CMT2M has an autosomal dominant inheritance pattern. DNM2 is a ubiquitously expressed large GTPase involved in both clathrin-dependent and -independent endocytosis and intracellular membrane trafficking. This protein has a strong interaction with actin and microtubule networks and may play a role in centrosome function. The initial symptoms of CMT2M start before the third decade of life. Although CMT2M overlaps in clinical features with intermediate CMTDIB, this type is purely axonal CMT. Distal muscle weakness and atrophy, distal sensory impairment, areflexia, and hyporeflexia can occur. The motor NCV value is in the low-to-normal range. Reduced myelin fibers, rare segmental demyelination/ remyelination, onion bulb formation, and axonal degeneration can be observed through nerve biopsy.

\section{CMT2N}

CMT2N is caused by a heterozygous mutation in the alanyltRNA synthetase (AARS) gene on chromosome 16q21 [43] which encodes the amino acid synthetases responsible for the initiation of the attachment of their respective amino acids to the corresponding tRNA. CMT2N has an autosomal dominant inheritance pattern. The onset of CMT2N ranges from 6 to 54 years, with fluctuating severity. Distal muscle weakness in the lower limbs mainly occurs. Additional symptoms include foot drop, ankle sprains, gait disturbance, foot deformities, distal sensory impairment, and hypo- or areflexia. Sensorineural hearing impairment has also been reported in a single household.

\section{CMT20}

CMT2O is caused by a heterozygous mutation in the dynein cytoplasmic 1 heavy chain 1 (DYNC1H1) gene on chromosome $14 q 32$ [44]. It has an autosomal dominant inheritancepattern. The DYNC1H1 gene encodes a large (>530 kDa) crucial subunit of the cytoplasmic dynein complex. Dyneins are a group of microtubule-activated ATPases that support the conversion of chemical energy into mechanical energy. CMT2O has been reported in only one family member. It begins during childhood and progresses slowly. Ambulation is usually maintained during adulthood. The main symptom of CMT2O is recurrent falls due to distal muscle weakness. Additional symptoms include pes cavus, distal sensory impairment, hyporeflexia, and neuropathic pain. Delayed motor development was observed, but learning difficulties were less frequent. The motor NCV is normal or mildly reduced. Axonal degenerative processes are revealed in 
sural nerve biopsies.

\section{CMT2P (autosomal dominant, autosomal recessive)}

CMT2P is caused by either a homozygous or heterozygous mutation in the leucine-rich repeat and sterile alpha motif-containing 1 (LRSAM1) gene on chromosome 9q33 [45]. LRSAM1 is a multifunctional RING finger protein that selectively regulates cell adhesion molecules, has ubiquitin ligase activity, and plays a role in receptor endocytosis and viral budding. The onset of CMT2P ranges from childhood to late adulthood, but usually occurs in adulthood. It progresses slowly. The initial symptoms are muscle weakness in the lower limbs. Those with CMT2P show difficulty in heel-to-toe gait and lose the ability to run. Fasciculations, muscle cramps, and foot deformities may also occur. Some patients become wheelchair-bound. Finally, a reduction in myelinated fibers is observed in the sural nerve biopsy.

\section{6. СMT20}

CMT2Q is caused by a heterozygous loss-of-function (LOF) mutation in the dehydrogenase E1 and transketolase domain containing 1 (DHTKD1) gene on chromosome 10p14 [46], as reported in a 5-generation Chinese family. CMT2Q has an autosomal dominant inheritance pattern. DHTHD1 is predicted to be a thiamine diphosphate-dependent 2-oxoacid dehydrogenase responsible for the reduction of the protein-bound $\mathrm{Li}^{+}$group. Muscle weakness of the distal lower limbs is the main symptom of CMT20. Atrophy of the distal forearms and hand intrinsic muscles, sensory impairment, and decreased deep tendon reflexes can also occur. In addition, disorganized myofilaments, small angulated muscle fibers, mitochondrial vacuolization, and sarcomere disappearance can be observed through muscle biopsy.

\section{CMT2R}

CMT2R is caused by either a homozygous or a compound heterozygous mutation in the tripartite motif containing 2 (TR/M2) gene on chromosome $4 q[47]$. CMT2R has an autosomal recessive inheritance pattern. TRIM2 functions as an E3 ubiquitin ligase that directs proteasome-mediated degradation of target proteins. The symptoms of CMT2R begin in early childhood. CMT2R causes distal muscle weakness and atrophy, especially atrophy of the hand and foot muscles. Additionally, an inability to walk on heels, broad-based gait, hypotonia, pes cavus, and pes equinovarus can be found. Specifically, one patient presented with knee contractures, respiratory insufficiency, tracheomalacia, and vocal cord paralysis. CMT2R shows slow motor NCV $(<30$ $\mathrm{m} / \mathrm{s}$ ) and decreased SNAP and CMAP amplitudes. In sural nerve biopsy, axonal degeneration, reduced myelinated fibers, accumulation of neurofilaments within axons, and swollen myelinated fibers can be found.

\section{CMT2S}

CMT2S is caused by either a homozygous or compound heterozygous mutation in the immunoglobulin mu DNA binding protein 2 (IGHMBP2) gene on chromosome 11q13 [48]. CMT2S has an autosomal recessive inheritance pattern. CMT2S occurs during the first decade of life. Although CMT2S progresses slowly, most patients are wheelchair-bound. The main symptoms of CMT2S are muscle weakness and sensory impairment, hypoor areflexia, foot drop, and steppage gait. Some patients may develop mild proximal muscle weakness. In addition, scoliosis, abnormal tongue shape, and pes equinovarus are also observed. In sural nerve biopsy, axonal degeneration, and reduced large myelinated fibers can be observed.

\section{CMT2T}

CMT2T is caused by either a homozygous or compound heterozygous mutation in the membrane metalloendopeptidase (MME) gene on chromosome 3q25 [49]. The MME gene encodes a widely expressed membrane metalloendopeptidase that degrades several substrates with its active site facing the extracellular space. CMT2T begins during late adulthood (between 36 and 56 years of age) and progresses slowly. The main features are distal muscle and sensory impairment, gait instability, foot drop, and hypo- or areflexia. Sural nerve biopsy reveals reduced large myelinated fibers.

\section{CMT2U}

$\mathrm{CMT} 2 \mathrm{U}$ is caused by a heterozygous mutation in the MARS gene on chromosome 12q13 [50]. CMT2U has an autosomal dominant inheritance pattern. CMT2U begins after the age of 50 with slow progression. Two unrelated families have also been reported. Muscle weakness and atrophy are the main features of the CMT2U. Additional symptoms include distal sensory impairment, decreased deep tendon reflexes, and steppage gait.

\section{CMT2V}

CMT2 $\mathrm{V}$ is caused by a heterozygous mutation in the $\mathrm{N}$-acetylalpha-glucosaminidase (NAGLU) gene on chromosome 17q21 [51]. CMT2V has an autosomal dominant inheritance pattern. The age of onset ranges from 18 to 61 years, which was found in a family from French-Canadian origin. Pain in the lower limbs 
and subsequent paresthesia in the upper limbs can occur. The additional common symptoms are tandem gait disturbances, and patients with sensory ataxia show sleep disturbances. Motor NCV was normal, while decreased SNAP and CMAP amplitudes were detected.

\section{CMT2W}

This type of CMT is caused by a heterozygous mutation in the histidyl-tRNA synthetase (HARS) gene on chromosome $5 q 31$ [52]. CMT2W has an autosomal dominant inheritance pattern. HARS catalyzes the covalent ligation of histidine to its cognate tRNA, representing an early step in protein biosynthesis. CMT2W begins widely during childhood to late adulthood, with highly fluctuating severity. CMT2W shows distal muscle weakness and lower limb atrophy, distal sensory impairment, steppage gait, hammer toes, pes cavus, and hypo- or areflexia. Finally, the motor NCV decreases slightly.

\section{CMT2X}

CMT2X is caused by either a homozygous or compound heterozygous mutation in the spatacsin vesicle trafficking associated (SPG11) gene on chromosome 15q21 [53]. CMT2X has an autosomal recessive inheritance pattern. The SPG11 gene encodes the protein spatacsin, which plays a role in axonal growth, function, and intracellular cargo trafficking. CMT2X begins at 4 to 35 years of age. CMT2X progresses slowly, and its severity varies widely. The main symptoms are distal muscle weakness and sensory impairment. Additional symptoms include decreased deep tendon reflexes, foot drop, gait disturbance, hand deformities, ankle contractures, kyphoscoliosis, and urinary dysfunction. CMT2X is also characterized by a thin corpus callosum and mild cognitive impairment. Finally, chronic denervation/reinnervation was observed using EMG. In addition, loss of large-caliber myelinated fibers has been observed through sural nerve biopsy.

\section{CMT2Y}

CMT2Y arises from a heterozygous mutation in the valosincontaining protein (VCP) gene on chromosome 9p13 [54], which encodes a ubiquitously expressed multifunctional protein, a member of the AAA+ (ATPase associated with various activities) protein family. CMT2Y has an autosomal dominant inheritance pattern. Only one family member and one unrelated patient have been reported. The onset age of CMT2Y varies from infancy to late adulthood ( $>50$ years), and its severity varies widely. Typically, the main symptom of CMT2Y is distal muscle weakness and atrophy of both the lower and upper extremities. Additional features include distal sensory impairment, difficulties with running and balance, and decreased deep tendon reflexes. Muscle biopsy confirmed neurogenic atrophy in one patient.

\section{CMT2Z}

CMT2Z is caused by a heterozygous mutation in the MORC family CW-type zinc finger 2 (MORC2) gene on chromosome $22 q 12[55,56]$. CMT2Z has an autosomal dominant inheritance pattern. MORC2 is a DNA-dependent ATPase that relaxes chromatin to ensure the repair of DNA double-strand breaks. Cytosolic MORC2 appears to be involved in lipid metabolism and homeostasis. The onset age of CMT2Z ranges from infancy to early adulthood. CMT2Z has a slow progression of disability. However, their severity varies widely, and some patients are limited to wheelchairs. The main symptom is asymmetric muscle involvement in the upper and lower limbs. Proximal muscle weakness, including neck flexion weakness, can also occur at a later stage of the disease. Distal sensory impairment, hyporeflexia, and pes cavus are frequently observed in patients. Pyramidal signs, developmental delays, and learning disabilities may also occur in this subtype. Other features include spontaneous muscle activity, muscle cramps, fasciculations, increased muscle tone, and myokymia. Furthermore, urinary incontinence, scoliosis, and claw hands are frequently observed in some patients. Hearing loss was observed in two members of the family. A high-pitched voice has been reported in another family. A sural nerve biopsy showed a loss of large myelinated fibers, while small onion bulbs and the variable presence of regenerative clusters are occasionally observed.

\section{Other CMT2}

HMSN-P is caused by TRK-fused gene (TFG) mutations, which can be found in a family of both Japanese and Korean populations $[57,58]$. CMT is usually characterized by distal muscle weakness, while HMSN-P is associated with proximal muscle weakness at a late onset of age. It shows a rapid progression of AF. Peripheral neuropathy, myopathy, hoarseness, and hearing loss (PNMHH) are caused by mutations in myosin heavy chain 14 (MYH14) [59-61] which occurs at a young age. Early symptoms include hearing impairment and muscle weakness, and mutations in the histidine triad nucleotide-binding protein 1 (HINT1) have been identified as the cause of autosomal recessive axonal neuropathy, which is mainly involved in neuroblastomas [62]. Finally, diacylglycerol 0-acyltransferase 2 (DGAT2), which catalyzes the final step of triglyceride biosynthesis, was observed in a Korean family with early onset dominance. It is character- 
ized by low triglyceride levels and sensory ataxia [63].

\section{Conclusion}

In this review, we identified the phenotypic characteristics of axonal CMT2 neuropathy. CMT is the most frequent disease among rare diseases, and in recent years, many therapeutic approaches have been developed. To date, more than 50 candidate therapeutic chemicals have been used in clinical trials or are being prepared for CMT. In addition, knowing the exact cause of genetic mutations in CMT patients can make it possible to give birth to a normal child through PGD. Currently, this is being practiced in CMT patients to become pregnant. Therefore, accurate diagnosis is essential for CMT patients. We hope that this review of the phenotypic characteristics of axonal CMT2 neuropathy will be of great help to clinicians majoring in medical genetics.

\section{Authors' Contributions}

Conception and design: BOC. Acquisition of data: HMK. Analysis and interpretation of data: all authors. Drafting the article: all authors. Critical revision of the article: BOC. Final approval of the version to be published: all authors.

\section{References}

1. Schröder JM. Neuropathology of Charcot-Marie-Tooth and related disorders. Neuromolecular Med 2006;8:23-42.

2. Harding $A E$, Thomas PK. The clinical features of hereditary motor and sensory neuropathy types I and II. Brain 1980;103:259-80.

3. Rudnik-Schöneborn S, Tölle D, Senderek J, Eggermann K, Elbracht M, Kornak $U$, et al. Diagnostic algorithms in Charcot-Marie-Tooth neuropathies: experiences from a German genetic laboratory on the basis of 1206 index patients. Clin Genet 2016;89:34-43.

4. Saporta AS, Sottile SL, Miller $\sqcup$, Feely SM, Siskind CE, Shy ME. Charcot-Marie-Tooth disease subtypes and genetic testing strategies. Ann Neurol 2011;69:22-33.

5. Rossor AM, Polke JM, Houlden H, Reilly MM. Clinical implications of genetic advances in Charcot-Marie-Tooth disease. Nat Rev Neurol 2013:9:562-71.

6. Tooth $\mathrm{HH}$. The peroneal type of progressive muscular atrophy $[\mathrm{PhD}$ dissertation]. London: H.K. Lewis; 1886.

7. Charcot JM, Marie P. Sur une forme particulière d'atrophie musculaire progressive souvent familiale débutant par les pieds et les jambes et atteignant plus tard les mains. Rev Med 1886;6:97-138.
8. Pareyson D, Marchesi C. Diagnosis, natural history, and management of Charcot-Marie-Tooth disease. Lancet Neurol 2009;8:654-67.

9. Horacek $O$, Mazanec $R$, Morris CE, Kobesova A. Spinal deformities in hereditary motor and sensory neuropathy: a retrospective qualitative, quantitative, genotypical, and familial analysis of 175 patients. Spine (Phila Pa 1976) 2007;32:2502-8.

10. Bamford NS, White KK, Robinett SA, Otto RK, Gospe SM Jr. Neuromuscular hip dysplasia in Charcot-Marie-Tooth disease type 1A. Dev Med Child Neurol 2009;51:408-11.

11. Werheid F, Azzedine $H_{1}$ Z Zwerenz E, Bozkurt A, Moeller MJ, Lin L, et al. Underestimated associated features in CMT neuropathies: clinical indicators for the causative gene? Brain Behav 2016;6:e00451.

12. Saifee TA, Pareés I, Kassavetis $P_{1}$ Kaski $D$, Bronstein AM, Rothwell JC, et al. Tremor in Charcot-Marie-Tooth disease: no evidence of cerebellar dysfunction. Clin Neurophysiol 2015;126:1817-24.

13. Anzalone $\mathrm{CL}$, Nuhanovic $\mathrm{S}$, Olund $\mathrm{AP}$, Carlson ML. Cochlear implantation in Charcot-Marie-Tooth disease: case report and review of the literature. Case Rep Med 2018;2018:1760978.

14. Lerat J, Magdelaine C, Beauvais-Dzugan H, Espil C, Ghorab K, Latour $P$, et al. A novel pathogenic variant of NEFL responsible for deafness associated with peripheral neuropathy discovered through nextgeneration sequencing and review of the literature. J Peripher Nerv Syst 2019;24:139-44.

15. Sambuughin N, de Bantel A, McWilliams S, Sivakumar K. Deafness and CMT disease associated with a novel four amino acid deletion in the PMP22 gene. Neurology 2003;60:506-8.

16. Nicholson G, Myers S. Intermediate forms of Charcot-Marie-Tooth neuropathy: a review. Neuromolecular Med 2006;8:123-30.

17. Papadakis CE, Hajiioannou JK, Kyrmizakis DE, Bizakis JG. Bilateral sudden sensorineural hearing loss caused by Charcot-Marie-Tooth disease. J Laryngol Otol 2003;117:399-401.

18. Zhao C, Takita J, Tanaka Y, Setou M, Nakagawa T, Takeda S, et al Charcot-Marie-Tooth disease type $2 \mathrm{~A}$ caused by mutation in a microtubule motor KIF1Bbeta. Cell 2001;105:587-97.

19. Reilly MM. Axonal Charcot-Marie-Tooth disease: the fog is slowly lifting! Neurology 2005;65:186-7.

20. Züchner S, Mersiyanova IV, Muglia M, Bissar-Tadmouri N, Rochelle $J$, Dadali EL, et al. Mutations in the mitochondrial GTPase mitofusin 2 cause Charcot-Marie-Tooth neuropathy type 2A. Nat Genet 2004;36:449-51.

21. Honda $S$, Aihara $T$, Hontani M, Okubo $K$, Hirose $S$. Mutational analysis of action of mitochondrial fusion factor mitofusin-2. J Cell Sci 2005;118(Pt 14):3153-61.

22. Nicholson GA, Magdelaine $C$, Zhu D, Grew S, Ryan MM, Sturtz F, et al. Severe early-onset axonal neuropathy with homozygous and compound heterozygous MFN2 mutations. Neurology 2008;70:1678-81. 
23. Verhoeven $K$, De Jonghe $P$, Coen $K$, Verpoorten $N$, Auer-Grumbach $M$, Kwon JM, et al. Mutations in the small GTP-ase late endosomal protein RAB7 cause Charcot-Marie-Tooth type 2B neuropathy. Am J Hum Genet 2003;72:722-7.

24. De Sandre-Giovannoli $A$, Chaouch $M$, Kozlov $S$, Vallat JM, Tazir M, Kassouri $\mathrm{N}$, et al. Homozygous defects in LMNA, encoding lamin A/ C nuclear-envelope proteins, cause autosomal recessive axonal neuropathy in human (Charcot-Marie-Tooth disorder type 2) and mouse. Am J Hum Genet 2002;70:726-36.

25. Leal $A$, Huehne $K$, Bauer $F$, Sticht $H$, Berger $P$, Suter $U$, et al. Identification of the variant Ala335Val of MED25 as responsible for CMT2B2: molecular data, functional studies of the $\mathrm{SH} 3$ recognition motif and correlation between wild-type MED25 and PMP22 RNA levels in CMT1A animal models. Neurogenetics 2009;10:275-87.

26. Klein CJ, Shi Y, Fecto F, Donaghy M, Nicholson G, McEntagart ME, et al. TRPV4 mutations and cytotoxic hypercalcemia in axonal CharcotMarie-Tooth neuropathies. Neurology 2011;76:887-94.

27. Klein CJ, Cunningham JM, Atkinson EJ, Schaid DJ, Hebbring SJ, Anderson SA, et al. The gene for HMSN2C maps to 12q23-24: a region of neuromuscular disorders. Neurology 2003;60:1151-6.

28. Rebelo AP, Abrams A, Cottenie E, Horga A, Gonzalez M, Bis DM, et al. Cryptic amyloidogenic elements in the 3' UTRs of neurofilament genes trigger axonal neuropathy. Am J Hum Genet 2016;98:597-614.

29. Brownlees J, Ackerley S, Grierson AJ, Jacobsen NJ, Shea K, Anderton $\mathrm{BH}$, et al. Charcot-Marie-Tooth disease neurofilament mutations disrupt neurofilament assembly and axonal transport. Hum Mol Genet 2002:11:2837-44.

30. Antonellis A, Ellsworth RE, Sambuughin N, Puls I, Abel A, Lee-Lin SO, et al. Glycyl tRNA synthetase mutations in Charcot-Marie-Tooth disease type $2 \mathrm{D}$ and distal spinal muscular atrophy type $\mathrm{V}$. Am J Hum Genet 2003;72:1293-9.

31. Lassuthova P, Rebelo AP, Ravenscroft G, Lamont PJ, Davis MR, Man-

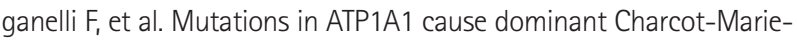
Tooth type 2. Am J Hum Genet 2018;102:505-14.

32. Carter J, Gragerov A, Konvicka K, Elder G, Weinstein H, Lazzarini RA. Neurofilament (NF) assembly; divergent characteristics of human and rodent NF-L subunits. J Biol Chem 1998;273:5101-8.

33. Irobi J, Van Impe K, Seeman P, Jordanova A, Dierick I, Verpoorten N, et al. Hot-spot residue in small heat-shock protein 22 causes distal motor neuropathy. Nat Genet 2004;36:597-601.

34. Barhoumi $C$, Amouri $R$, Ben Hamida C, Ben Hamida M, Machghoul $S$, Gueddiche $M$, et al. Linkage of a new locus for autosomal recessive axonal form of Charcot-Marie-Tooth disease to chromosome 8q21.3. Neuromuscul Disord 2001;11:27-34.

35. De Jonghe $P$, Timmerman $V$, Ceuterick $C$, Nelis $E$, De Vriendt $E$, Löfgren A, et al. The Thr124Met mutation in the peripheral myelin protein zero (MPZ) gene is associated with a clinically distinct CharcotMarie-Tooth phenotype. Brain 1999;122(Pt 2):281-90.

36. Birouk N, Azzedine H, Dubourg O, Muriel MP, Benomar A, Hama-

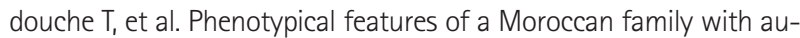
tosomal recessive Charcot-Marie-Tooth disease associated with the S194X mutation in the GDAP1 gene. Arch Neurol 2003;60:598-604.

37. Marrosu MG, Vaccargiu S, Marrosu G, Vannelli A, Cianchetti $C_{1}$ Muntoni F. Charcot-Marie-Tooth disease type 2 associated with mutation of the myelin protein zero gene. Neurology 1998;50:1397-401.

38. Senderek J, Hermanns B, Lehmann U, Bergmann C, Marx G, Kabus C, et al. Charcot-Marie-Tooth neuropathy type 2 and PO point mutations: two novel amino acid substitutions (Asp61Gly; Tyr119Cys) and a possible "hotspot" on Thr124Met. Brain Pathol 2000;10:235-48.

39. Claramunt R, Pedrola L, Sevilla T, López de Munain A, Berciano J, Cuesta $A$, et al. Genetics of Charcot-Marie-Tooth disease type 4A: mutations, inheritance, phenotypic variability, and founder effect. J Med Genet 2005;42:358-65.

40. Chung KW, Kim SM, Sunwoo IN, Cho SY, Hwang SJ, Kim J, et al. A novel GDAP1 Q218E mutation in autosomal dominant CharcotMarie-Tooth disease. J Hum Genet 2008;53:360-4.

41. Tang BS, Zhao GH, Luo W, Xia K, Cai F, Pan O, et al. Small heat-shock protein 22 mutated in autosomal dominant Charcot-Marie-Tooth disease type 2L. Hum Genet 2005;116:222-4.

42. Fabrizi GM, Ferrarini M, Cavallaro T, Cabrini I, Cerini R, Bertolasi L, et al. Two novel mutations in dynamin-2 cause axonal Charcot-MarieTooth disease. Neurology 2007;69:291-5.

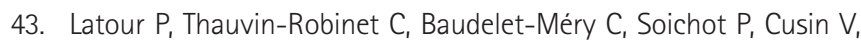
Faivre $L$, et al. A major determinant for binding and aminoacylation of tRNA(Ala) in cytoplasmic Alanyl-tRNA synthetase is mutated in dominant axonal Charcot-Marie-Tooth disease. Am J Hum Genet 2010;86:77-82.

44. Weedon MN, Hastings R, Caswell R, Xie W, Paszkiewicz K, Antoniadi $T_{1}$ et al. Exome sequencing identifies a DYNC1H1 mutation in a large pedigree with dominant axonal Charcot-Marie-Tooth disease. Am J Hum Genet 2011;89:308-12.

45. Weterman $M A$, Sorrentino $V_{1}$ Kasher $P R$, Jakobs $M E$, van Engelen $B G$, Fluiter $K_{1}$ et al. A frameshift mutation in LRSAM1 is responsible for a dominant hereditary polyneuropathy. Hum Mol Genet 2012;21:35870.

46. Xu WY, Gu MM, Sun LH, Guo WT, Zhu HB, Ma JF, et al. A nonsense mutation in DHTKD1 causes Charcot-Marie-Tooth disease type 2 in a large Chinese pedigree. Am J Hum Genet 2012;91:1088-94.

47. Ylikallio E, Pöyhönen R, Zimon M, De Vriendt E, Hilander T, Paetau A, et al. Deficiency of the E3 ubiquitin ligase TRIM2 in early-onset axonal neuropathy. Hum Mol Genet 2013;22:2975-83.

48. Cottenie $E_{1}$ Kochanski $A$, Jordanova $A$, Bansagi $B$, Zimon $M$, Horga $A$ 
et al. Truncating and missense mutations in IGHMBP2 cause CharcotMarie Tooth disease type 2. Am J Hum Genet 2014;95:590-601.

49. Higuchi Y, Hashiguchi A, Yuan J, Yoshimura A, Mitsui J, Ishiura $H_{\text {, et }}$ al. Mutations in MME cause an autosomal-recessive Charcot-MarieTooth disease type 2. Ann Neurol 2016;79:659-72.

50. Gonzalez M, McLaughlin H, Houlden H, Guo M, Yo-Tsen L, Hadjivassi-

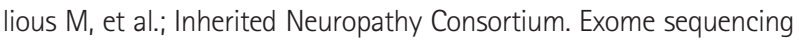
identifies a significant variant in methionyl-tRNA synthetase (MARS) in a family with late-onset CMT2. J Neurol Neurosurg Psychiatry 2013;84:1247-9.

51. Tétreault M, Gonzalez M, Dicaire MJ, Allard P, Gehring K, Leblanc D, et al. Adult-onset painful axonal polyneuropathy caused by a dominant NAGLU mutation. Brain 2015;138(Pt 6):1477-83.

52. Vester A, Velez-Ruiz G, McLaughlin HM; NISC Comparative Sequenc-

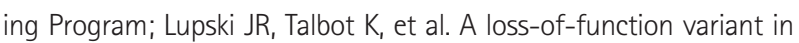
the human histidyl-tRNA synthetase (HARS) gene is neurotoxic in vivo. Hum Mutat 2013;34:191-9.

53. Montecchiani C, Pedace L, Lo Giudice T, Casella A, Mearini M, Gaudi-

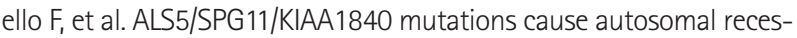
sive axonal Charcot-Marie-Tooth disease. Brain 2016;139(Pt 1):73-85.

54. Gonzalez MA, Feely SM, Speziani F, Strickland AV, Danzi M, Bacon C, et al. A novel mutation in VCP causes Charcot-Marie-Tooth type 2 disease. Brain 2014;137(Pt 11):2897-902.

55. Albulym OM, Kennerson ML, Harms MB, Drew AP, Siddell AH, Auer-

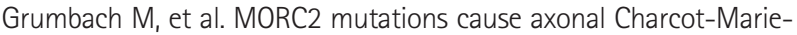
Tooth disease with pyramidal signs. Ann Neurol 2016;79:419-27.

56. Takashima H, Nakagawa M, Nakahara K, Suehara M, Matsuzaki T, Hi- guchi I, et al. A new type of hereditary motor and sensory neuropathy linked to chromosome 3. Ann Neurol 1997;41:771-80.

57. Ishiura H, Sako W, Yoshida M, Kawarai T, Tanabe O, Goto J, et al. The TRK-fused gene is mutated in hereditary motor and sensory neuropathy with proximal dominant involvement. Am J Hum Genet 2012;91:320-9.

58. Lee SS, Lee HJ, Park JM, Hong YB, Park KD, Yoo JH, et al. Proximal dominant hereditary motor and sensory neuropathy with proximal dominance association with mutation in the TRK-fused gene. JAMA Neurol 2013;70:607-15.

59. Choi BO, Kang SH, Hyun YS, Kanwal S, Park SW, Koo H, et al. A complex phenotype of peripheral neuropathy, myopathy, hoarseness, and hearing loss is linked to an autosomal dominant mutation in MYH14. Hum Mutat 2011;32:669-77.

60. Iyadurai S, Arnold WD, Kissel JT, Ruhno C, Mcgovern VL, Snyder PJ, et al. Variable phenotypic expression and onset in MYH14 distal hereditary motor neuropathy phenotype in a large, multigenerational North American family. Muscle Nerve 2017;56:341-5.

61. Almutawa W, Smith C, Sabouny R, Smit RB, Zhao T, Wong R, et al. The R941 L mutation in MYH14 disrupts mitochondrial fission and associates with peripheral neuropathy. EBioMedicine 2019;45:379-92.

62. Zimoń M, Baets J, Almeida-Souza L, De Vriendt E, Nikodinovic J, Parman $Y$, et al. Loss-of-function mutations in HINT1 cause axonal neuropathy with neuromyotonia. Nat Genet 2012;44:1080-3.

63. Hong YB, Kang J, Kim JH, Lee J, Kwak G, Hyun YS, et al. DGAT2 mutation in a family with autosomal-dominant early-onset axonal Charcot-Marie-Tooth disease. Hum Mutat 2016;37:473-80. 